\title{
INFLUENCE OF ROTATED GROUND MOTION COMPONENTS ON THE RESPONSE DISTRIBUTION OF INELASTIC OSCILLATORS
}

\author{
Dimitrios G. Giannopoulos ${ }^{1}$, and Dimitrios I. Vamvatsikos ${ }^{1}$ \\ ${ }^{1}$ National Technical University of Athens \\ Iroon Polytechneiou 9, Athens, Greece \\ e-mail: giandimpapaki@hotmail.com, divamva@mail.ntua.gr
}

Keywords: Incremental Dynamic Analysis, Angle of Incidence, Probabilistic Risk Assessment, Performance-Based Earthquake Engineering, Nonlinear Dynamic Analysis.

\begin{abstract}
Recent research has shown that structures may experience quite different response when the components of any given ground motion record are rotated in the horizontal plane. On the other hand, significant differences are also associated with using different ground motion records. This paper examines whether it is preferable to use a small set of records, each rotated to multiple incidence angles, or a larger set with fewer (or no) rotations, in the case we wish to perform a fixed number of nonlinear dynamic analyses and we have little site information. To this purpose we collected a relatively large, non-pulsive set of records and applied it to an elastoplastic single-degree-of-freedom (SDOF) system. Finally, bootstrapping was performed to investigate the effect of the number of rotation angles studied versus the number of records on the statistics of response. The results indicate that, in all cases, and especially when a small number of nonlinear analyses is allocated, the effect of record-torecord variability clearly outweighs the incidence angle influence. Using a small set of records can lead to unreliable results by both inaccurate estimation of the central value and severe underestimation of the dispersion.
\end{abstract}




\section{INTRODUCTION}

In the past few years, several researchers have examined the impact of the record orientation on both single-degree-of-freedom (SDOF) and multi-degree-of-freedom (MDOF) systems. As a general conclusion, it can be said that the incidence angle can influence the structural response. On the other hand, various studies have shown that when performing time history analyses, e.g. with Incremental Dynamic Analysis (IDA, Vamvatsikos and Cornell [1]), the use of large sets of records is crucial, for the better probabilistic assessment of the results, due to the substantial record-to-record variability.

Particularly, Rigato and Medina [2] studied the influence of the earthquake direction on seismic demand for both symmetrical and asymmetrical inelastic single-story structures, of various degrees of inelasticity and of a wide range of fundamental periods, and demonstrated that applying bi-directional ground motions only along the principal axes of an inelastic building underestimates the inelastic peak deformation demand when compared to those obtained at other angles of incidence. The same trend, with an increase of up to $37 \%$, for rotated records, in terms of both roof displacements and plastic hinge rotations, was found in the research of Magliulo et al [3], which was based on an L-plan shape multi-story RC building. Kalkan and Kwong [4] examined the merits and demerits of rotating ground motion records to fault-normal (FN) and fault-parallel (FP) for response history analysis in the case of pulsive records, using an instrumented 6-story concrete building. They concluded that though the critical angle varies with the ground motion pair and the response quantity of interest, the use of the larger response among the two values corresponding to the FN and FP directions, compared to no rotation at all, is warranted. Lagaros [5] implemented multicomponent incremental dynamic analysis (MIDA) on both symmetric and non-symmetric, in plan, 3D mid-rise RC buildings, while taking the incidence angle as variable. Based on 15 different records and on Latin Hypercube Sampling (LHS) he came to the conclusion that the use of 30 recordincidence angle pairs is sufficient for probabilistic analysis. The same author [6] researched the impact of the earthquake orientation on the seismic loss estimation in order to perform life cycle cost assessment using MIDA. He concluded that MIDA represents a more suitable way to take into consideration the randomness of both record and incidence angle, while his findings that 5 randomly chosen incident angles are enough for taking the randomness of attack of the earthquake into account, were based on an increasing number of total analyses. In the same study he highlighted that the use of a large number of different records is crucial. Fragiadakis et al [7] pointed up that different ground motion records, stemming from either different seismic events or even different recording stations, will produce different demand that varies considerably. Therefore, in order to get reliable response estimates, an appropriately selected set of several ground motion records is necessary, since a single time history analysis is of limited practical use. The uncertainty in the collapse fragility curve and furthermore in the mean annual frequency of collapse associated with record-to-record variability was examined by Eads et al [8] and it was shown that using a small number of ground motions can lead to unreliable estimates of a structure's collapse risk. Finally, we need to mention that using the seismic codes at their lower limit (of 3 or 7 records), has been the source of criticism for potentially unconservative results by several researchers (e.g. Bradley, [9]).

The objective of this paper is to investigate the effect of the number of both rotations and records on the structural response, based on a statistical approach, and suggest whether it is preferable to use a small set of records, each rotated to various angles, or a larger set of records, without considering more orientations, in the case we can only run a fixed number of analyses and there is little site information that would favor one orientation over another. As a first step we present plots which make clear that both rotations and records can severely influ- 
ence the elastic and inelastic response of a SDOF system and then we give the full IDA and statistical analyses for SDOF systems of various periods for the non-pulsive set of records. Based on the aforementioned, it will be shown that the record-to-record variability clearly overshadows the impact of the incidence angle, especially when a small number of non-linear dynamic analyses is conducted.

\section{A FIRST APPROACH TO ROTATION-TO-ROTATION AND RECORD-TO- RECORD VARIABILITY}

Initially we examine the influence of both rotations and records in the case of an elastic SDOF system that was arbitrarily chosen to have a period of $1 \mathrm{sec}$. This model is analyzed for 30 different records, each one rotated with a constant step of $1^{\circ}$ in the interval of $\left[0^{\circ}, 180^{\circ}\right]$, and the maximum elastic displacement versus the angle of rotation is shown in Figure 1. From this plot, it is clear that the angle of rotation affects the maximum response of the structure, since every single line, which corresponds to a different record, takes different values while the orientation $\theta$ changes. It is also observed that for $\theta=0^{\circ}$, we have a vast range of values for the elastic displacement, which means that different records lead to different responses as well.

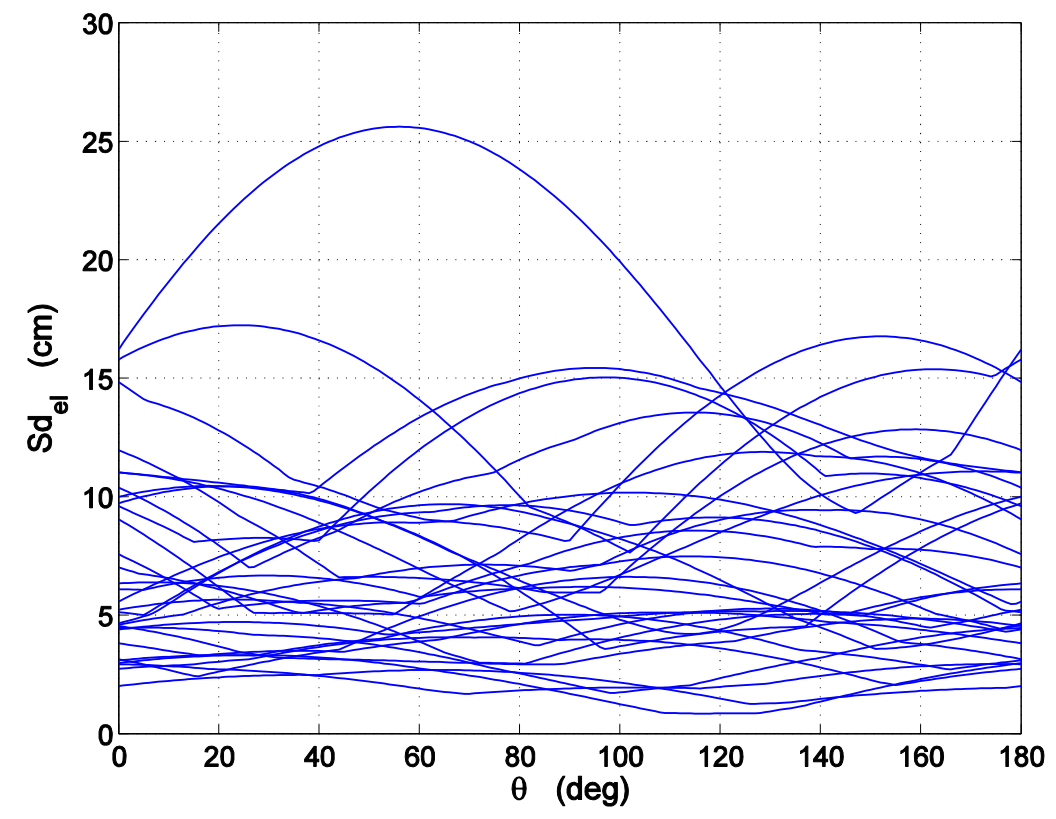

Figure 1: Maximum elastic displacement for 30 records versus angle of rotation. $T=1 \mathrm{sec}, \zeta=5 \%$.

The records used for Figure 1 were of different intensity, as they were applied as originally recorded. On top of that, in real life all structures are designed to yield and reach their plastic region, so the approach based on the response of the elastic SDOF system is insufficient. In order to address this problem, we create the elastic response spectra shown in Figures 2a and $2 \mathrm{~b}$, for $\zeta=5 \%$, which are all scaled to the same value of spectral-acceleration for $T=1$ sec. Particularly, Figure 2a shows 30 spectra of the same record (based on the event of Loma Prieta) rotated with a constant step of $6^{\circ}$ in the interval $\left[0^{\circ}, 180^{\circ}\right.$ ), while Figure $2 \mathrm{~b}$ depicts 30 spectra each one from a different recording. In the case of a SDOF system with period $T=1$ sec, yielding would cause a period larger than $1 \mathrm{sec}$, clearly leading to uncertainty in terms of structural response as it is evident in both figures for periods greater than $1 \mathrm{sec}$. Thus, it is obvious that the structural response is a function of both orientation and record, due to the fact that for periods greater than the initial fundamental period, the seismic demand takes values from a wide range. 


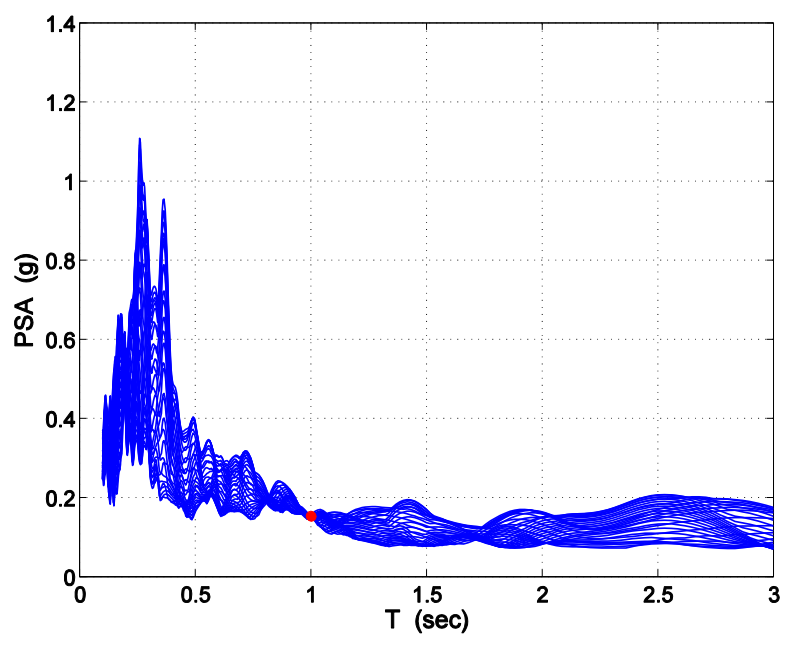

(a) Same record, different orientations.

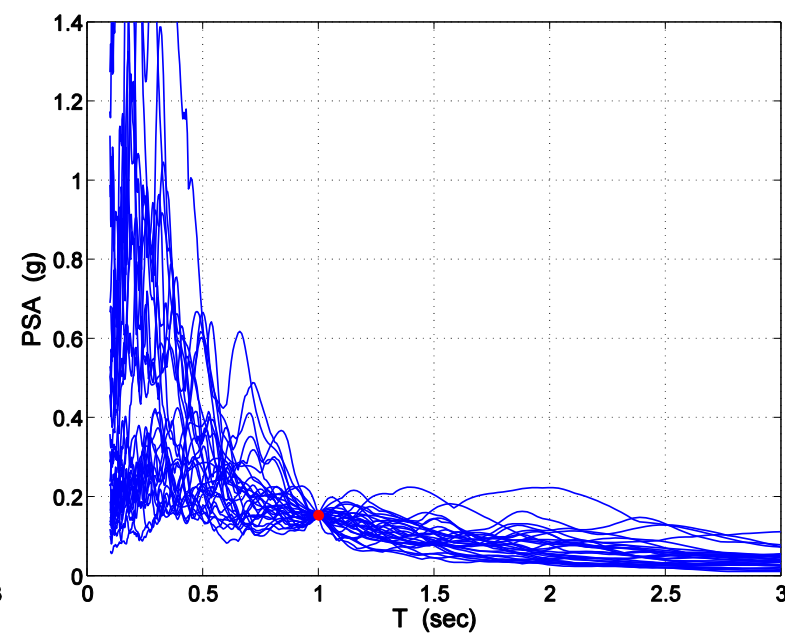

(b) One orientation, different records.

Figure 2: Scaled $(T=1 \mathrm{sec})$ elastic response spectra for $\zeta=5 \%$ for (a) various orientations of the same record and (b) various unrotated records. Both figures show 30 spectra.

Thus far, it has been shown that both records and rotations seriously affect the seismic demand. In order to quantify the emerging uncertainty and obtain an idea on which variable is more critical, we implement statistical methods, and particularly bootstrapping [11], on a large number of IDA curves of a SDOF system with $T=1 \mathrm{sec}$, that originate from 135 records and 60 rotation angles, i.e. $135 \times 60=8,100$ total analyses. The details regarding these dynamic analyses, the probabilistic assessment methods, the set of records, the SDOF model etc, will be given in the next chapter. We make the assumption that the total number of analyses is constant and equal to 60 . That being said, we use two statistical approaches: one with 60 different records for each sample, therefore only 1 angle of rotation, and one with 2 different records for each sample, thus 30 equidistant angles of rotation. From the analyses mentioned before we create Figures $3 \mathrm{a}$ and $3 \mathrm{~b}$. Figure $3 \mathrm{a}$ shows the plasticity $\mu$, defined as the maximum plastic displacement over the yielding displacement, versus the normalized intensity $R$, defined as the demand acceleration over the yield acceleration. On Figure $3 \mathrm{~b}$ we plot the dispersion $\beta$ of each sample, defined as the standard deviation of the logarithm of the data, versus the normalized intensity. From Figure $3 a$ it is apparent that when using a small number of records, the mean of the median value of all samples is close enough to the actual median value that is taken from the whole population. When using only one random sample, which is the case when designing a real structure, the probability of having a large deviation that would lead to unrealistic results, either conservative or unconservative, is much higher than in the case of using a larger number of records. From Figure $3 \mathrm{~b}$ it is apparent that choosing to use few records rotated in various orientations will lead in both underestimation, in means of central value, of the natural dispersion which is caused by the phenomenon of the earthquake itself, as it is clear by comparing the mean value of all samples with the one of the whole population, and increased estimation error due to the poor sampling. This error is obviously downsized when using a large number of records. From all the previous analyses, it is clear that for this SDOF model the use of a large number of different records will produce much more realistic and reliable results than using a large number of angles of incidence, due to the fact that the record-to-record variability is greater than the rotation-to-rotation one. In the following chapters we investigate whether this trend stands for various values of the period of the SDOF system. 


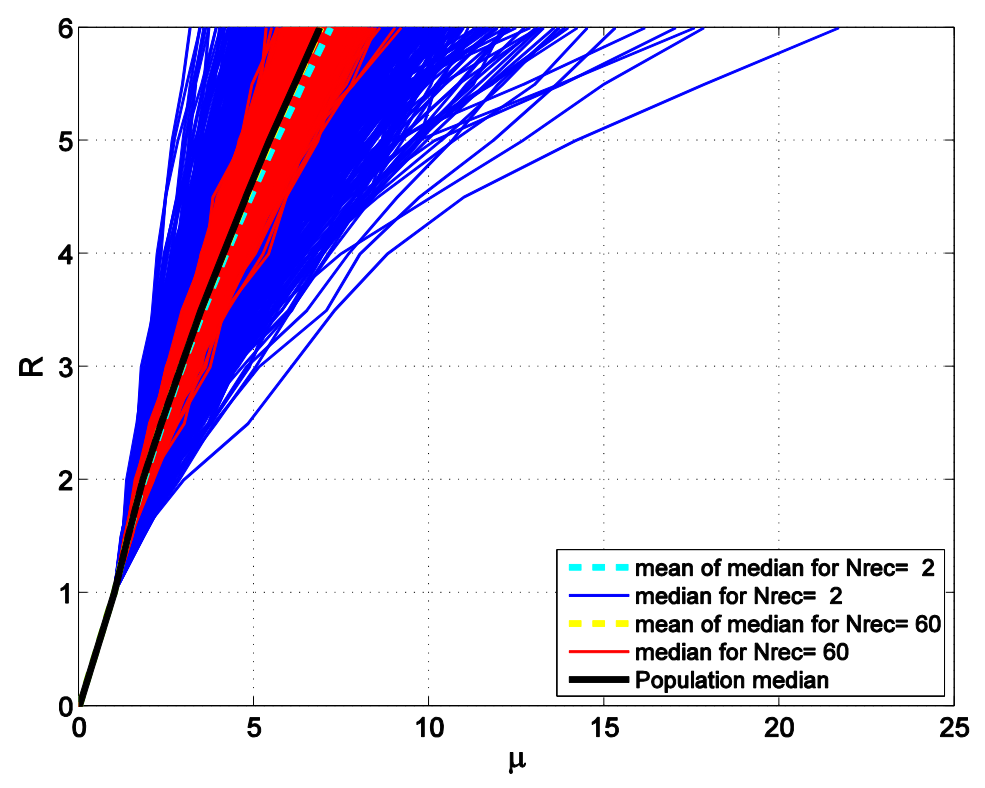

(a) Distribution of the median.

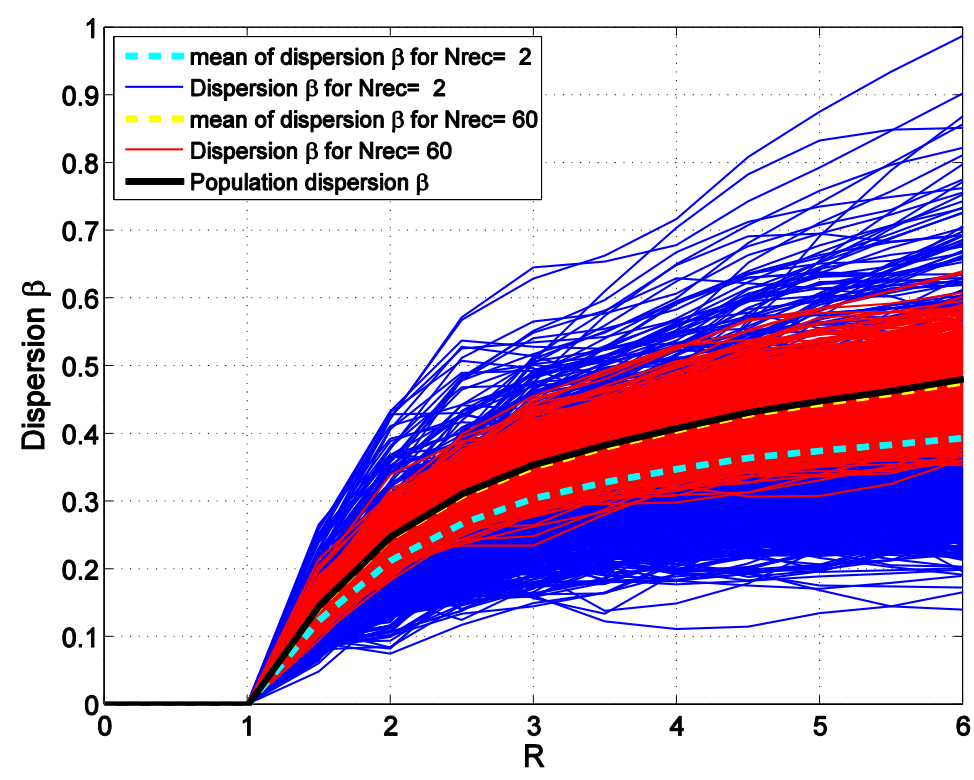

(b) Distribution of the dispersion.

Figure 3: Statistical distribution of a SDOF model with $T=1 \mathrm{sec}$ and $\zeta=5 \%$, for (a) Median and (b) dispersion, for a total of 60 analyses.

\section{ANALYSIS OF SDOF MODEL WITH NON-PULSIVE SET OF RECORDS}

\subsection{Assumptions, input data and basic calculations}

The SDOF system examined is a relatively simple model. Particularly, we assume that the system is bilinear with elastic-perfectly plastic response, while degradation and hardening have not been taken into account. The damping ratio is $5 \%$ and we use a wide range of periods, i.e. $0.2,0.5,1.0$ and $2.0 \mathrm{sec}$, so as to investigate both rigid and flexible systems. The yielding displacement is arbitrarily assumed to be $d_{y}=0.035 \mathrm{~m}$. 
Regarding the earthquake data, we use a set of 135 non-pulsive records from 33 events all around the world from all kinds of faults. The maximum number of records from each earthquake was chosen to be equal to 8 to avoid introducing any bias, caused by over-representing a single event. The moment magnitude ranges from 6.4 to 7.7, while the Joyner-Boore distance ranges from 0 to $30 \mathrm{Km}$. Despite the small distance, care was exercised to avoid "consensus" pulse-like motions. The site class, according to the NEHRP, is chosen to be C or D, so the soil shear wave velocity takes values from 183 to $762 \mathrm{~m} / \mathrm{s}$.

The as-recorded accelerograms are rotated in the horizontal plane using the equations:

$$
\begin{aligned}
& x_{a}=a_{x} \cos \theta+a_{y} \sin \theta \\
& y_{a}=-a_{x} \sin \theta+a_{y} \cos \theta
\end{aligned}
$$

$\theta$ being the angle of rotation in the counterclockwise direction, with a constant step of $3^{\circ}$ in the $\left[0^{\circ}, 180^{\circ}\right)$ interval, creating 60 different orientations. For the scaling of the accelerograms that is needed to conduct IDA's, we define the normalized intensity $R$ in the interval $[1.5,6]$ with a constant step of 0.5 , thus every IDA has 10 levels of intensity.

Finally, for the statistical assessment of the results we use bootstrap. Particularly, we create a large number of samples $(10,000)$ for each case and the results produced by this sampling can be assumed to represent the potential variation for the statistical parameters of interest. Based on this methodology, we can easily come to conclusions regarding whether it is better to use more orientations or more records by comparing both central values and dispersions.

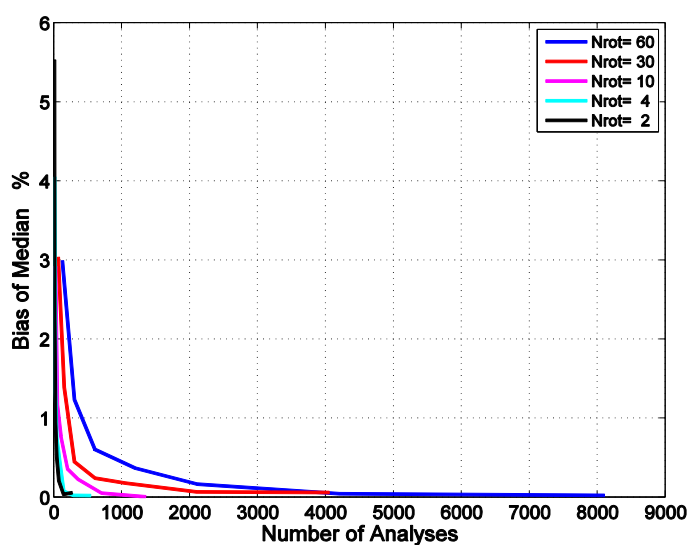

(a) Bias of the median

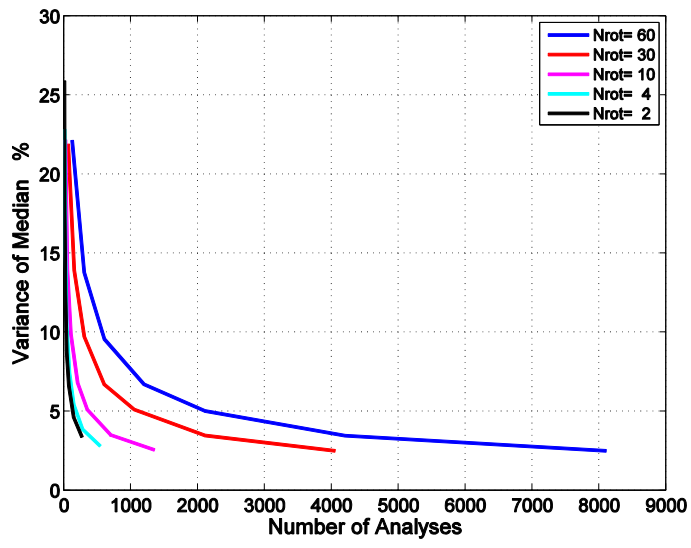

(c) Variance of the median

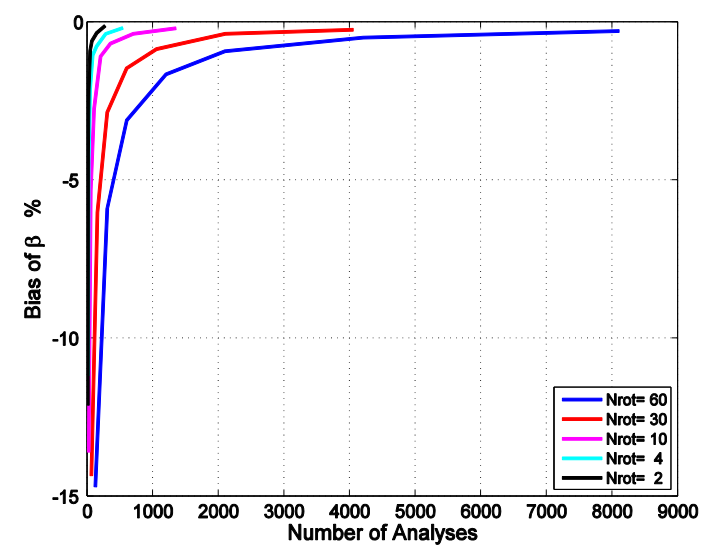

(b) Bias of the dispersion $\beta$

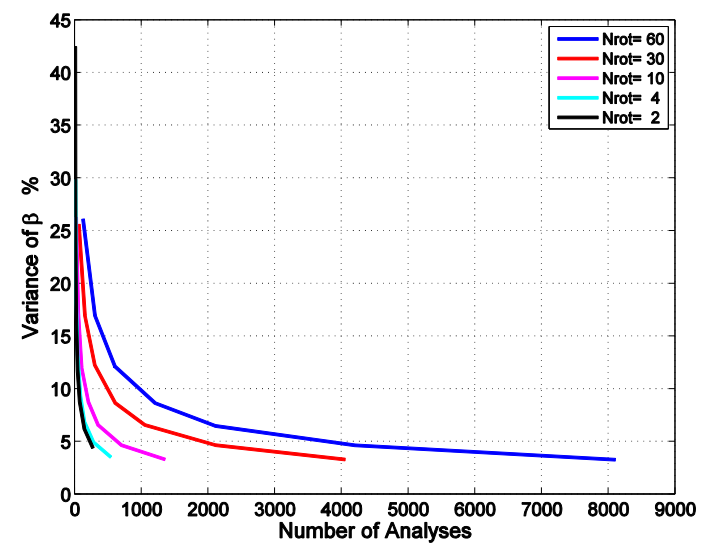

(d) Variance of the dispersion $\beta$

Figure 4: Constant number of rotations for $T=1 \mathrm{sec}$ (a) Bias of the median, (b) Bias of the dispersion $\beta$, (c) Variance of the median and (d) Variance of the dispersion $\beta$. 


\subsection{Results}

After running the analyses for the whole set of records, i.e. $135 \times 60=8,100$ analyses, for each period mentioned before, we choose to use two comparison paradigms: the first one is for constant number of rotations while the number of total analyses, i.e. the number of records, is increasing and the second one is for constant number of total analyses while both numbers of rotations and records are variables. In the next paragraphs we give the results for $T=1 \mathrm{sec}$ and $T=0.2 \mathrm{sec}$ as the results for $T=2 \mathrm{sec}$ and $T=0.5 \mathrm{sec}$ follow the same trends.

Using the approach of constant number of rotations, we take the graphs shown in Figures 4 and 5. From these figures it is clear that: i) for constant number of total analyses, the more records we use the less error we have in the results, ii) regardless of the number of rotations, the increase in the number of records leads to decreased values of estimation error, iii) the increase in the number of rotations does not decrease the error, iv) by using few records for longer periods, the bias of the median is negligible, while the bias of the dispersion as well as the variance of both the median and the dispersion are not, meaning that by choosing only one single sample for real life applications both the median and the dispersion may include severe errors leading to unreal estimates of the collapse probability and v) by using few records for lower periods, the error in the estimates is much higher and cannot be neglected even for the central value of the median.

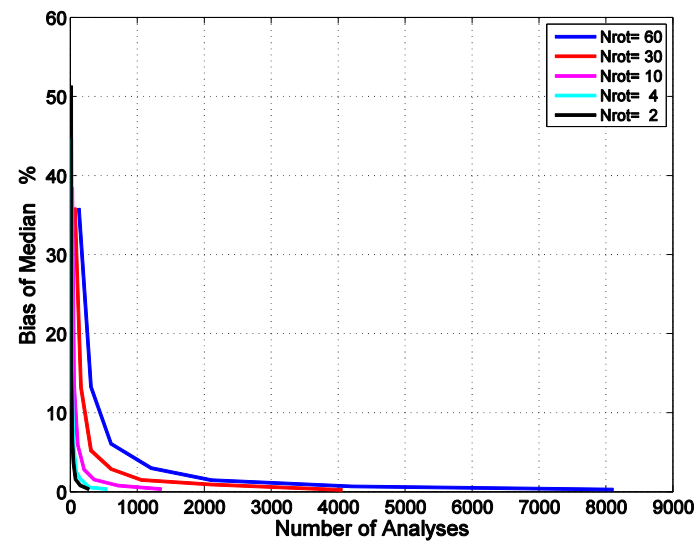

(a) Bias of the median

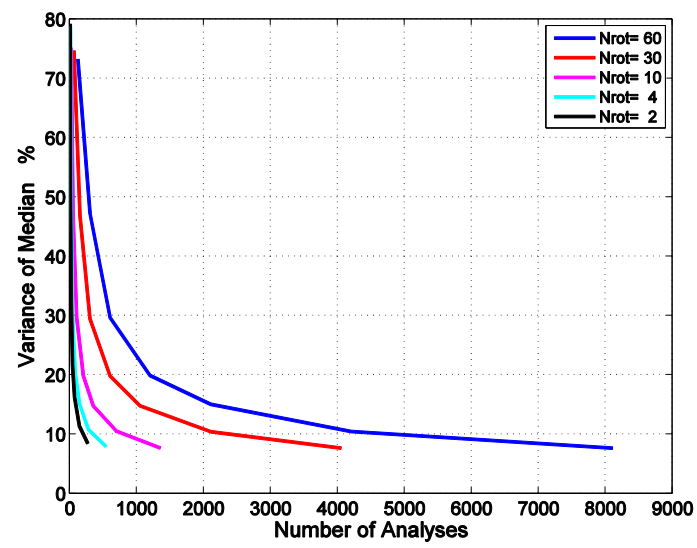

(c) Variance of the median

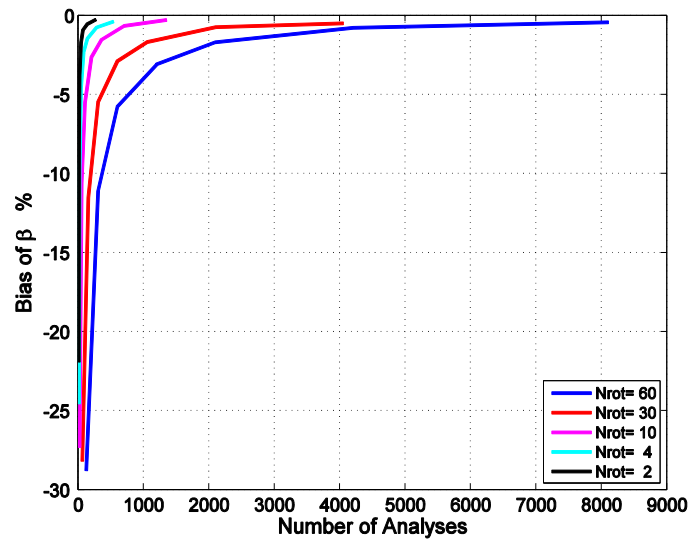

(b) Bias of the dispersion $\beta$

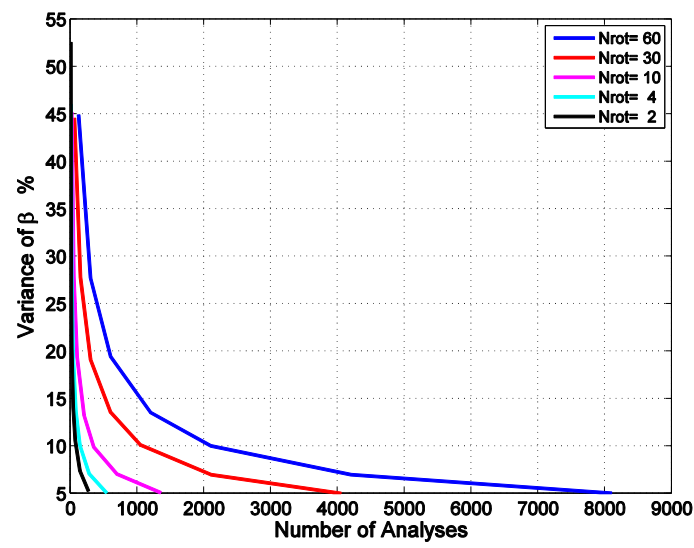

(d) Variance of the dispersion $\beta$

Figure 5: Constant number of rotations for $T=0.2 \mathrm{sec}$ (a) Bias of the median, (b) Bias of the dispersion $\beta$, (c) Variance of the median and (d) Variance of the dispersion $\beta$. 


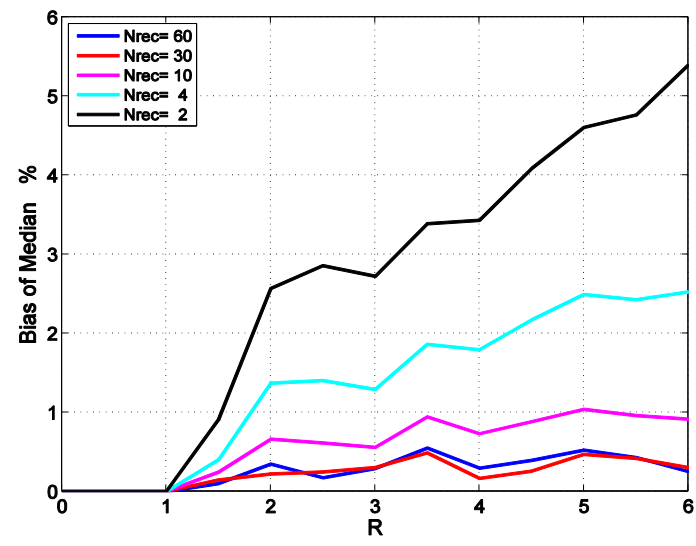

(a) Bias of the median

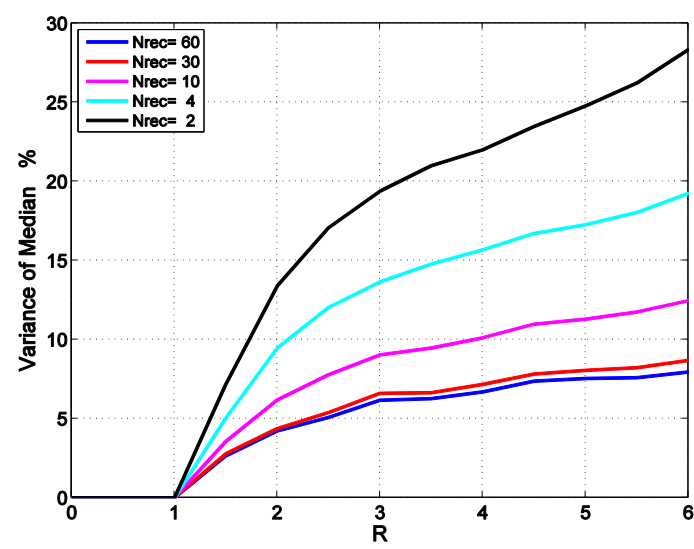

(c) Variance of the median

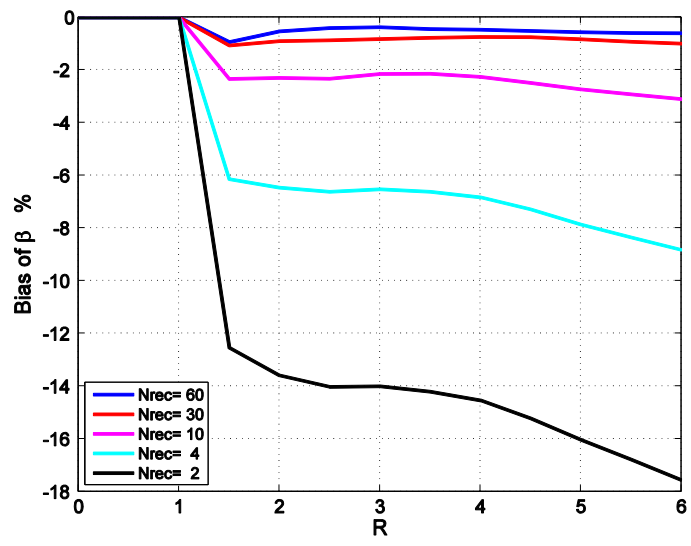

(b) Bias of the dispersion $\beta$

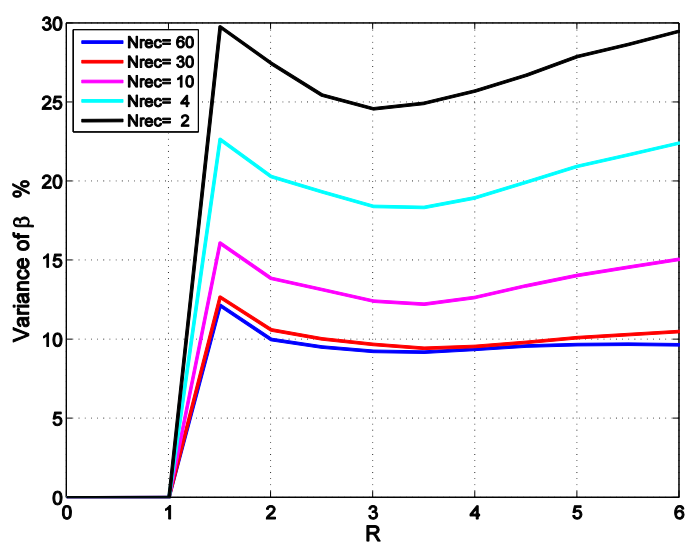

(d) Variance of the dispersion $\beta$

Figure 6: Constant number of analyses Ntot $=60$ for $T=1 \mathrm{sec}$ (a) Bias of the median, (b) Bias of the disper$\operatorname{sion} \beta$, (c) Variance of the median and (d) Variance of the dispersion $\beta$.

Using the approach of constant number of analyses for Ntot $=60$ we take the graphs shown in Figures 6 and 7. From these figures we see that: i) the more records used the less error is introduced, ii) for longer periods the bias of the central value tends to be zero regardless of the number of records used, but all the other parameters have severe errors, iii) the error increases for lower periods and iv) the differences between 30 and 60 records are negligible meaning that using 30 records can lead to acceptable results for an elastoplastic SDOF; meanwhile 10 records may lead to wrong estimates so the provisions of the EC8 for using either the maximum response based on 3 records or the mean based on 7 can lead to unconservative calculations.

In the aforementioned, when using the term error we mean the bias and the variance of the statistical parameter being examined. Particularly, the bias is defined as the difference between the mean value of the parameter (median or dispersion $\beta$ ) based on all samples and the actual parameter value taken from the whole population over the latter, while the variance is defined as the coefficient of variation, i.e. the standard deviation over the mean of the parameter. 


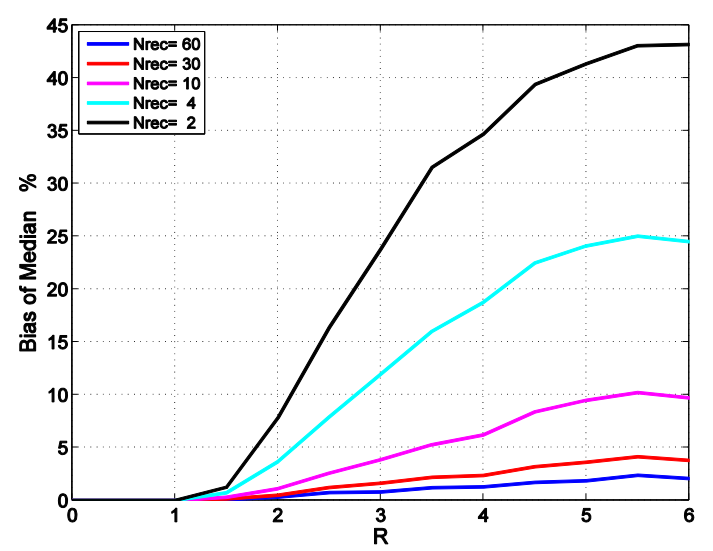

(a) Bias of the median

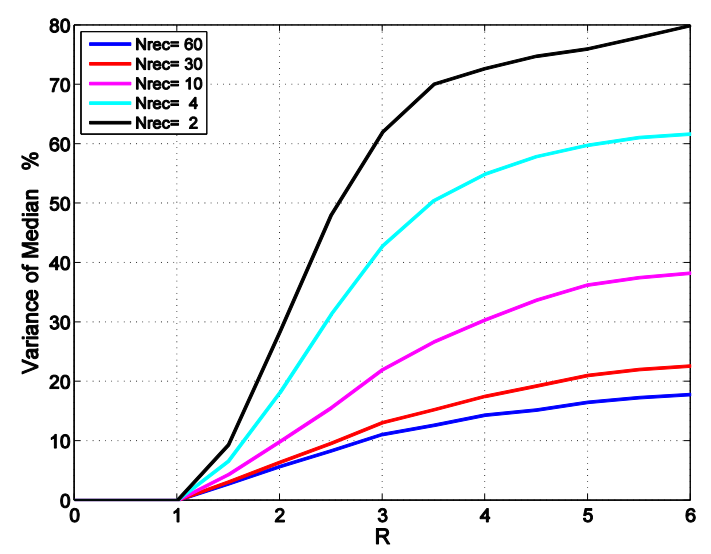

(c) Variance of the median

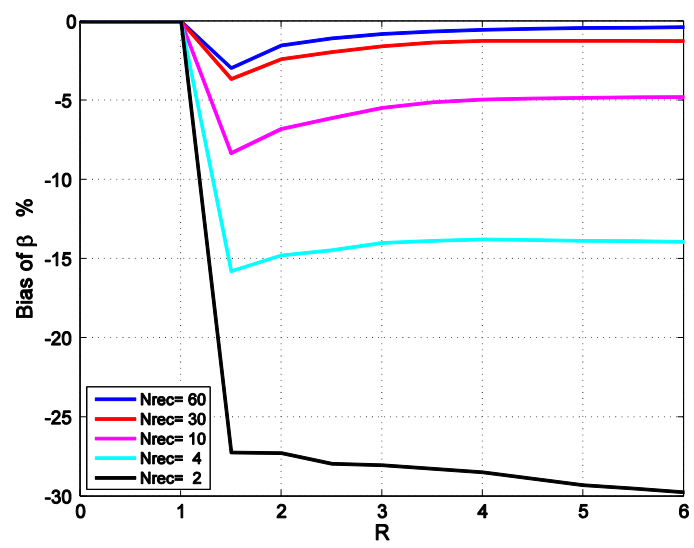

(b) Bias of the dispersion $\beta$

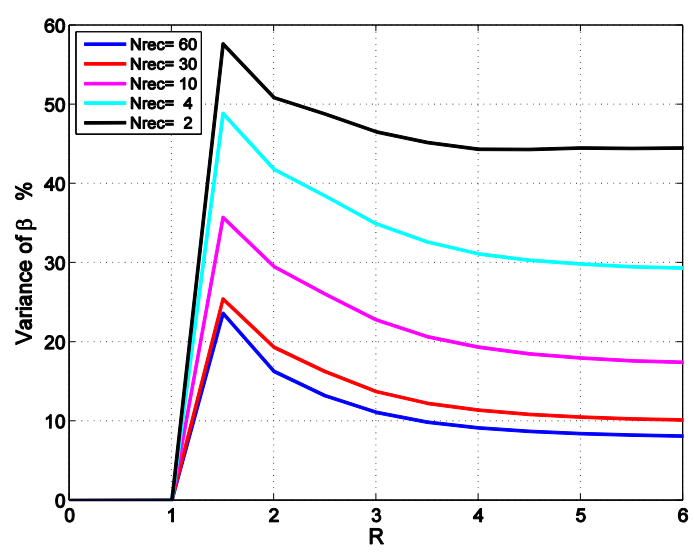

(d) Variance of the dispersion $\beta$

Figure 7: Constant number of analyses $N_{\text {tot }}=60$ for $T=0.2 \mathrm{sec}$ (a) Bias of the median, (b) Bias of the disper$\operatorname{sion} \beta$, (c) Variance of the median and (d) Variance of the dispersion $\beta$.

\section{CONCLUSIONS}

The effect of the incidence angle on the structural response has been investigated in probabilistic terms vis-à-vis the number of distinct records used while running time history analyses. The most significant findings of this work can be summarized as follows:

- The record-to-record variability is clearly larger than the one of rotation-to-rotation, so, in the case of having no site information regarding the actual orientation of the fault with respect to the structure, the use of the largest set of records possible is warranted.

- The provisions of EC8 regarding time history analysis that suggest using the maximum response based on 3 records or the mean based on 7 records can lead to unconservative results, especially if one is interested in the dispersion of structural demand. The use of the central value based on 30 records seems to be accurate enough.

- The error induced by poor sampling when using a small number of records is larger for lower periods of the oscillator. 
We need to mention that these findings only concern a single-degree of freedom oscillator. Still, we expect that real structures will generally show similar results, unless perhaps there is some directional preference in the structural system. This subject is the current research topic of the authors.

\section{REFERENCES}

[1] D. Vamvatsikos, C.A. Cornell, Incremental dynamic analysis. Earthquake Engineering \& Structural Dynamics, 31(3), 491-514.

[2] A.B. Rigato, R.A. Medina, Influence of angle of incidence on seismic demands for inelastic single-storey structures subjected to bi-directional ground motions. Engineering Structures, 29, 2593-2601, 2007.

[3] G. Magliulo, G. Maddaloni, C. Petrone, Influence of earthquake direction on the seismic response of irregular plan RC frame buildings. Earthquake Engineering and Engineering Vibration, 13, 243-256, 2014.

[4] E. Kalkan, N.S. Kwong, Pros and cons of rotating ground motion records to faultnormal/parallel directions for response history analysis of buildings. Journal of Structural Engineering, 140, 04013062, 2014.

[5] N.D. Lagaros, Multicomponent incremental dynamic analysis considering variable incident angle. Structure and Infrastructure Engineering, 6, 77-94, 2010.

[6] N.D. Lagaros, The impact of the earthquake incident angle on the seismic loss estimation. Engineering Structures, 32, 1577-1589, 2010.

[7] M. Fragiadakis, D. Vamvatsikos, M. Karlaftis, N.D. Lagaros, M. Papadrakakis, Seismic assessment of structures and lifelines. Journal of Sound and Vibration, 334, 29-56, 2015.

[8] L. Eads, E. Miranda, H. Krawinkler, D.G. Lignos, An efficient method for estimating the collapse risk of structures in seismic regions. Earthquake Engineering \& Structural Dynamics, 42, 25-41, 2013.

[9] B.A. Bradley, Design seismic demands from seismic response analyses: A probabilitybased approach. Earthquake Spectra, 27(1), 213-224, 2011.

[10] A.K. Chopra, Dynamics of Structures: Theory and Applications to Earthquake Engineering, 4th Edition. Prentice Hall, 2012.

[11] B. Efron, R. Tibshirani. An Introduction to the Bootstrap. Chapman \& Hall, 1993. 